\title{
Foreword: Special Issue on Database Theory
}

\author{
Wim Martens ${ }^{1}$
}

Published online: 10 January 2018

(C) Springer Science+Business Media, LLC, part of Springer Nature 2018

This issue of Theory of Computing Systems contains five invited journal articles that extend papers that were presented at the International Conference on Database Theory (ICDT 2016), which was held jointly with the International Conference on Extending Database Technology (EDBT 2016) on March 15-18, 2016 in Bordeaux, France. ICDT is one of the major conferences for database theory. The ICDT conference series started in 1986 and was a biennial conference until 2009, when it became an annual conference and is jointly held with EDBT.

The submissions for the special issue underwent thorough journal reviewing and revision processes, in accordance with the usual standards of Theory of Computing Systems. The papers in this special issue are (in no particular order):

- Complexity and Expressive Power of Weakly Well-Designed SPARQL, by Mark Kaminski and Egor V. Kostylev.

- It's All a Matter of Degree: Using Degree Information to Optimize Multiway Joins, by Manas Joglekar and Christopher Ré.

- Document Spanners: From Expressive Power to Decision Problems, by Dominik D. Freydenberger and Mario Holldack.

- Limits of Schema Mappings, by Phokion G. Kolaitis, Reinhard Pichler, Emanuel Sallinger, and Vadim Savenkov.

- Reasoning About Integrity Constraints for Tree-Structured Data, by Wojciech Czerwiński, Claire David, Filip Murlak, and Paweł Parys.

I am very grateful to the authors and the reviewers for all their hard work, without which this special issue would not have been possible.

This article is part of the Topical Collection on Special Issue on Database Theory

Wim Martens

wim.martens@uni-bayreuth.de

1 Universität Bayreuth, Universitätsstraße 30, 95447 Bayreuth, Germany 